\title{
DEVELOPMENT OF AN EXPERT SYSTEM FOR REDUCING MEDICAL ERRORS
}

\author{
Mohamed Ramadan and Khalid Al-Saleh \\ Industrial Engineering Department, College of Engineering, King Saud University
}

\begin{abstract}
Recent advances in patient safety have been hampered by the hard dealing with the development of a uniform classification of patient safety concepts in a systematic way. Therefore, many believe that medical expert systems have great potential to improve health care. A framework for computer-based medical errors diagnoses of primary systems' deficiencies is presented. Results of this research assisted in developing the hierarchical structure of the medical errors expert system which was written and complied in CLIPS. It has 225 rules, 52 parameters and 830 conditional paragraphs. The system prompts the user for response with suggested input formats. The system checks the user input for consistency within the given limits. In addition, the system was validated through numerous consultations with the experts in the field. The benefits that are gained from such types of expert systems are eliminating the fear from dealing with personal mistake, and providing the up-date information and helps medical staff as a learning tool.
\end{abstract}

\section{KEYWORDS}

Medical errors, expert system, errors classification, patient safety \& medical errors diagnoses.

\section{INTRODUCTION}

In the medical field all kinds of errors are significant, and can have potentially dramatic effects $[1,2]$. It was found that the incidence of error included and affected all types of medical professionals at different stages of administration of medical care. This includes physicians, physician's assistants, pharmacists, nurses, and administrative personnel [3]. A complex relationship exists between medical errors and contributing factors. The same medical error or circumstance may be perceived as a medical error or a contributing factor, depending on the context, circumstance or outcome [4]. A medical error always has a set of contributing factors. Although a medical error can be a contributing factor to the origin or development of another incident, some contributing factors can not be incidents in their own right $[5,6]$. Research shows that most medical errors are largely preventable [7-9]. Harvard University conducted a study on medical errors, which led to the recognition that engineers specializing in human factors and health systems are needed to improve the existing system [10].

To reduce medical errors, identification and classification of errors must be stated in a cleared statement [11]. The identification and classification of medical errors in the medical setting is a very complicated process, and this process may be simplified by implementing an effective classification system $[12,13]$. In order to reach a consensus on the classification of medical errors, it is necessary to develop a generally accepted international medical error classification system [14]. Findings have indicated that errors are likely to affect patients in similar ways in countries with similar healthcare systems [15]. With this taxonomy, the major types of errors can be categorized and each major type can then be associated with a specific underlying mechanism. DOI : $10.5121 /$ ijsea.2013.4603 
This can explain why and even predict when and where an error will occur [16], which in turn will assist in the generation of intervention strategies for each type of error, and it will assist in the reduction of medical errors [17-19].

Expert systems are software systems that can be compared to human experts. Their purpose is mostly advisory. Besides, they give explanation and advice to human experts when performing certain tasks. They are intelligent information systems, and are capable to explain and justify their conclusions [20]. There are several types of problems that can be solved using knowledge-based systems [21-26]. Durkin [27] listed different application areas, including business, which encompasses marketing, management, finance, accounting, medical, etc. for doing control, design, diagnosis, instruction, interpretation, monitoring, planning, prediction, prescription, selection, detection, evaluation, computation, and classification.

In addition to the expert systems that have applications in different areas of medicine, a variety of medical expert systems tools are available and can function as intelligent assistants to clinicians, helping in diagnostic processes, laboratory analysis, treatment protocol, and teaching of medical students and residents [28-33]. Expert systems also have certain bad features such as they can not examine a patient instead of physicians. Finally, expert system that is good for one certain field is often not good for another one. They may confuse a physician and make him/her commit wrong decisions practically under time stress.

The primary goal of this research is to develop expert system applying recent medical errors classification and adverse events that are encountered in current medical practice to present some advice that might help to prevent those medical errors.

\section{BACKGROUND}

Many patient safety-related classifications were developed all over the world [6]. They were built using different techniques and contain different concepts, definitions and terms. Internationally, the agreed definition of patient safety concepts and a uniform approach to classify the concepts hinders comparison of information, learning and system improvement

Currently the World Health Organization's World league for patient safety has engaged in a project to develop an International Classification for Patient Safety "ICPS" to develop a classification system, which converts patient safety information collected from different systems into a common format to simplify aggregation, analysis and learning across fields, boundaries and times.

Knowledge-based expert systems employ human knowledge to solve problems that normally would require human expertise. These expert systems represent the expertise knowledge as data or rules within the computer. These rules and data can be called upon when needed to solve problems. Books and manuals have a huge amount of knowledge but a human has to read and interpret the knowledge for it to be utilized. Most of the expert systems are developed via specialized software tools called shells. These shells come equipped with an inference mechanism (backward chaining, forward chaining, or both), and require knowledge to be entered according to a specified format (all of which might lead some to categorize CLIPS ver. 6.3 [34] as a shell). Usually they appear with a number of other features, such as tools for writing hypertext, for building friendly user interfaces, for manipulating lists, strings, and objects, and for connecting with external programs and databases. These shells populate as languages, although certainly with a limited range of application than most programming languages. For more detailed information on expert system shells, see the "Expert System Shells at Work" series by Schmuller [35]. 


\section{DEVELOPING PREVENTIVE MEDICAL ERRORS EXPERT SYSTEM}

Based on the above literature, errors in the medical field can occur in many different ways, with potentially diverse, wide-ranging and hazardous effects. A review of the varied errors related to fundamental areas such as medication, diagnosis, treatment procedures and clerical procedures in terms of their number, etiology and possible ramifications, is a complex domain. Consideration of the number of possible alterations of specific elements or actions in a health-related setting that might be identified as error(s), connected with the large variety of "system" type errors, drives us to conclude that we are dealing with a tremendous range of possibilities.

A classification is an arrangement of concepts into classes and their subdivisions linked to express the semantic relationships between them. For example, 'contributing factors' precede and perform a role in the generation of any 'incident type' (see, Figure 1). An incident can be a reportable circumstance, near miss, or harmful incident (adverse event). A reportable circumstance is a situation in which there was significant chance for harm, but no incident occurred (i.e., a busy intensive care unit remaining grossly understaffed during all shift time, or taking a defibrillator to an emergency and finding it out does not work although it was not needed). A near miss is an incident which did not reach the patient (e.g., a unit of blood being connected to the wrong patient's vascular, but the error was discovered before the infusion started). A no harm incident is one in which an event reached a patient but no discernible harm resulted (e.g., if the unit of blood was infused, but was compatible). A harmful incident is an incident that results in harm to a patient (e.g., the wrong unit of blood was infused and the patient died from a haemolytic reaction).

Incidents are classified into a number of several types. An incident type is a class made up of incidents of a widespread nature, grouped because of shared agreed features and is a "parent" class under which many ideas may be grouped. For example, an incident in which an infusion pump was set up wrongly and delivered a sedative overdose, causing respiratory arrest, would be allocated both 'medication' and 'equipment' incident types. Incident types include clinical administration, clinical procedure, documentation, healthcare-associated infection, medication, blood, blood products, nutrition, oxygen, gas, vapour, medical device, medical equipment, behavior, patient accidents, infrastructure fixtures, resources and organizational management [6].

Expert System shell CLIPS is used to design this system. The system classifies errors based on a set of decision rules. Rule-based programming is one of the most commonly used techniques for developing expert systems. In the programming paradigm, rules are used as heuristics, which specify a set of actions to be executed and performed for a given situation. In the event that two rules match a given problem situation, the system will employ a conflict resolution strategy to best resolve the tie based on the specified decision rules. For example, it could break a tie based on which rule is more specific, or which rule is shorter or based on "refreshing" that is, rules, which had recently been done after conflict resolution, might not be used again for some time in prejudice of new rules. 


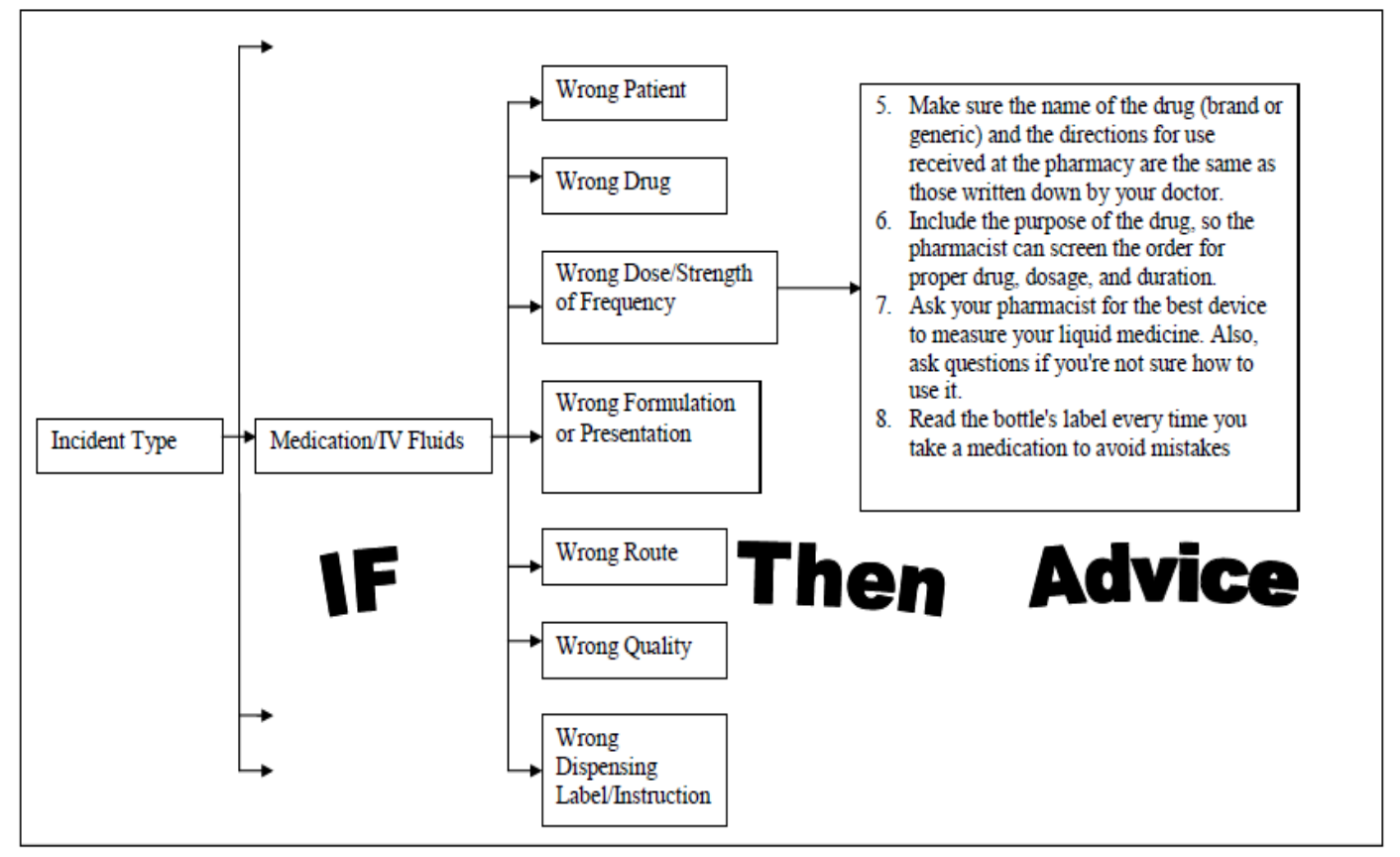

Figure 1. Part of the Network of Classifications of Medical Errors.

Actions taken to eliminate hazard are actions taken to eliminate and manage any future harm, or probability of harm, associated with an incident. Such actions can influence incidents, contributing factors, and can be pro-active or reactive. Pro-active actions may be identified by methods such as failure mode and effects analysis [36, 37] and probabilistic risk analysis [16], whereas reactive actions are taken in answering to insights obtained after incidents (e.g. root cause analysis). Resilience refers to the degree to which a system constantly prevents, detects, alleviates or improves risks or incidents. Resilience allows an organization to get back to its original ability to supply main functions as soon as possible after incurring harm (injury) [38].

The hierarchical structure of the expert system has been fully developed. It consists of a main program which is written and complied in CLIPS. It has 225 rules, 52 parameters and 830 conditional paragraphs of advice. The main sources of information for the development of the knowledge base were taken from different recourses [6, 12, 39-48].

The medical error knowledge domain is based on a variety of rules and utilizes CLIPS shell from NASA [34]. The knowledge base contains all information that the shell expert system can display a given material, as well as the information needed to construct and decide the displayed advice. All notifications were collected through consultations with medication staff from Tabouk area, an area in northern Saudi Arabia, as well as from Kopec et al. [49], Miller [50], and Halbach and Sullivan [51]. The data-base sections were divided into control, explanation, and advice logic rules. The purpose of control logic rules is to control where the consultation is and where will it go next. In addition, it assigns the values of user answers to the data base variables. An example of such rules is:

Rule \# 71

IF incident type $=$ Healthcare Associated Infection

AND 
International Journal of Software Engineering \& Applications (IJSEA), Vol.4, No.6, November 2013

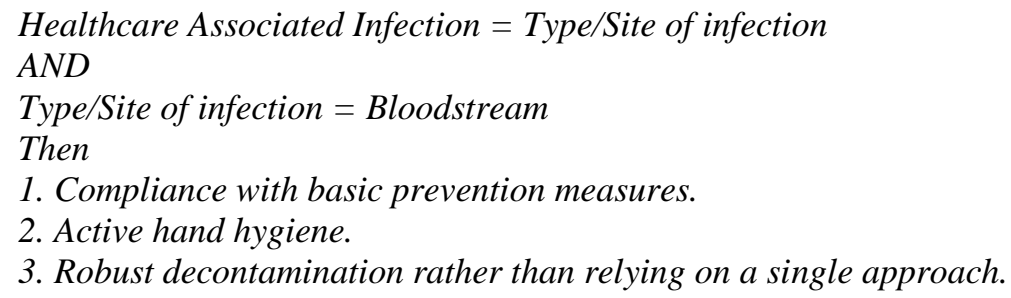

The explanation logic rules are designed messages at any time the users press how or why keys, or help when they are confused to understand the given question. The following example is a sample of the explanation rules:

"Human error in medical practice can be reduced if a culture of awareness emphasizing when and how errors are prone to occur, in part by implementing Expert System models, is affected. The general nature of human error(s) in complex systems is reviewed and focused on issues raised by Institute of Medicine, $1999^{1}$. From this background categorized error $(s)$ in medical practice, including medication, procedures, diagnosis, clerical error(s), and others are classified the World Health Organization, 2009 ${ }^{2}$. The primary goal of this developed Expert System is to identify and classify medical errors that are encountered in current medical practice. In addition, we would like to make recommendations for the design of a new type of "preventative system" that will introduce a new "gold" standard for medical systems. This includes physicians, physician's assistants, pharmacists, nurses, administrative personnel, and patient”.

The advice rules express the conclusion that has been reached through the consultation execution such as:

Recommendations of clinical process-procedure problems

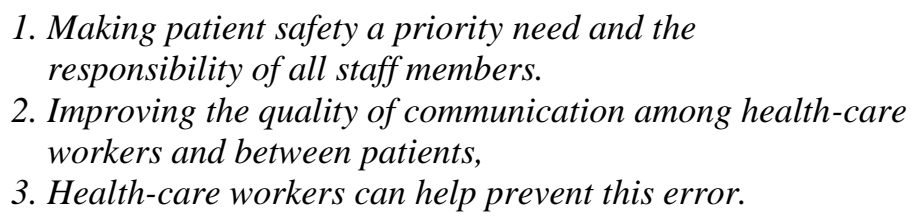

The system prompts the user for response with suggested input formats. The system checks the user input for consistency within the given limits. In addition, the system provides a brief explanation when the user is confused about particular prompt. Subsequently, the system was validated through numerous consultations with the experts in the field.

\section{RESULTS}

\section{Consultation Session}

Figure 2 shows the information presented as inputs and outputs that appear to the user during a consultation session with the developed expert system. First, the Medical Errors Clips software should be run using the CLIPS software version 6.3 provided from NASA [34]. Then, the user clicks on the "execution" command and "run" command in order to run the program.

Now the Expert System is ready for the consultation. If a medication incident exist and countermeasure for such type of incident are needed. Then the user needs to type "medication" and press 
Enter key. Figure 3 will then appear; and one of the causes of medication incident may be typed such as "wrong drug", for example. Enter key must then be pressed again. A window showing the expert recommendations will be displayed as shown in Figure 4. At the end of this session, the expert system may be run again by just clicking on the "execution" command and "run" command in order to run the program again.

\section{DISCUSSIONS AND CONCLUSIONS}

Results of this research assisted in developing the hierarchical structure of an expert system which consists of main program written and complied in CLIPS. The system prompts the user for response with suggested input formats. The system checks the user input for consistency within the given limits. In addition, the system provides a brief explanation if the user is confused about particular prompt. The period of knowledge acquisition lasted about 9 months; development of data bases required 5 months. Subsequently, the proposed medical errors expert system was evaluated and validated through numerous consultations with the experts in the field testing.

This study achieved its goal by developing expert system software to prevent medical errors. This study represents a continuous learning and improvement cycle emphasizing risk identification, prevention, detection, and reduction; incident recovery and system resilience. The developed expert system provides actions that should be taken to reduce risk. These actions concentrate on steps taken to prevent the reoccurrence of the same or similar patient safety incident and on improving system resilience. Actions taken to reduce hazard are those actions taken to minimize and control the hazard, or the probability of harm associated with an incident. These actions may be directed toward the patient (e.g., decision support), toward staff (e.g., training, availability of policies/procedures), toward the organization (e.g., improved leadership and guidance, proactive hazard assessment), and toward therapeutic agents and equipment (e.g., regular audits).

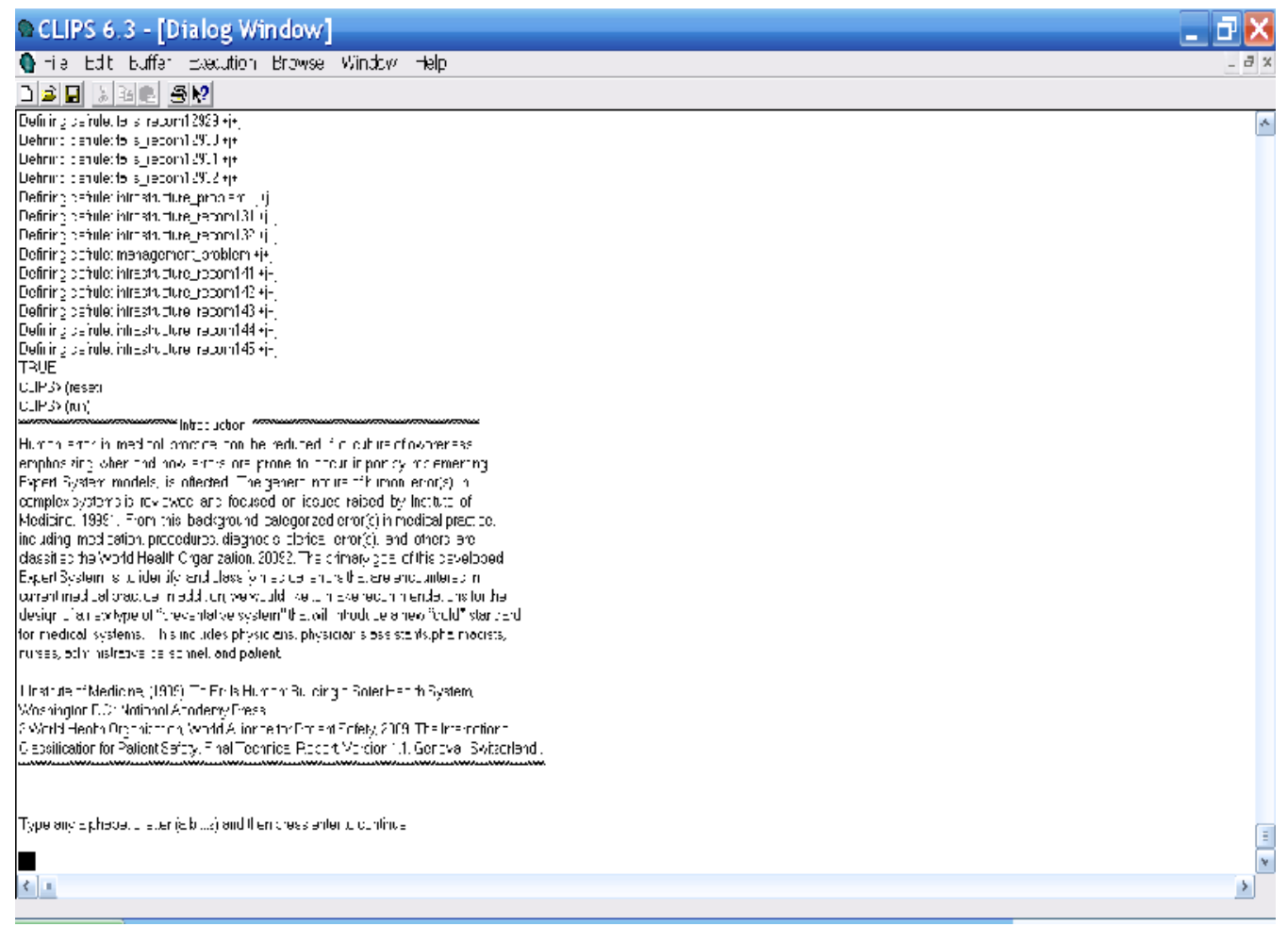


Figure 2. Appearance of starting expert system screen.

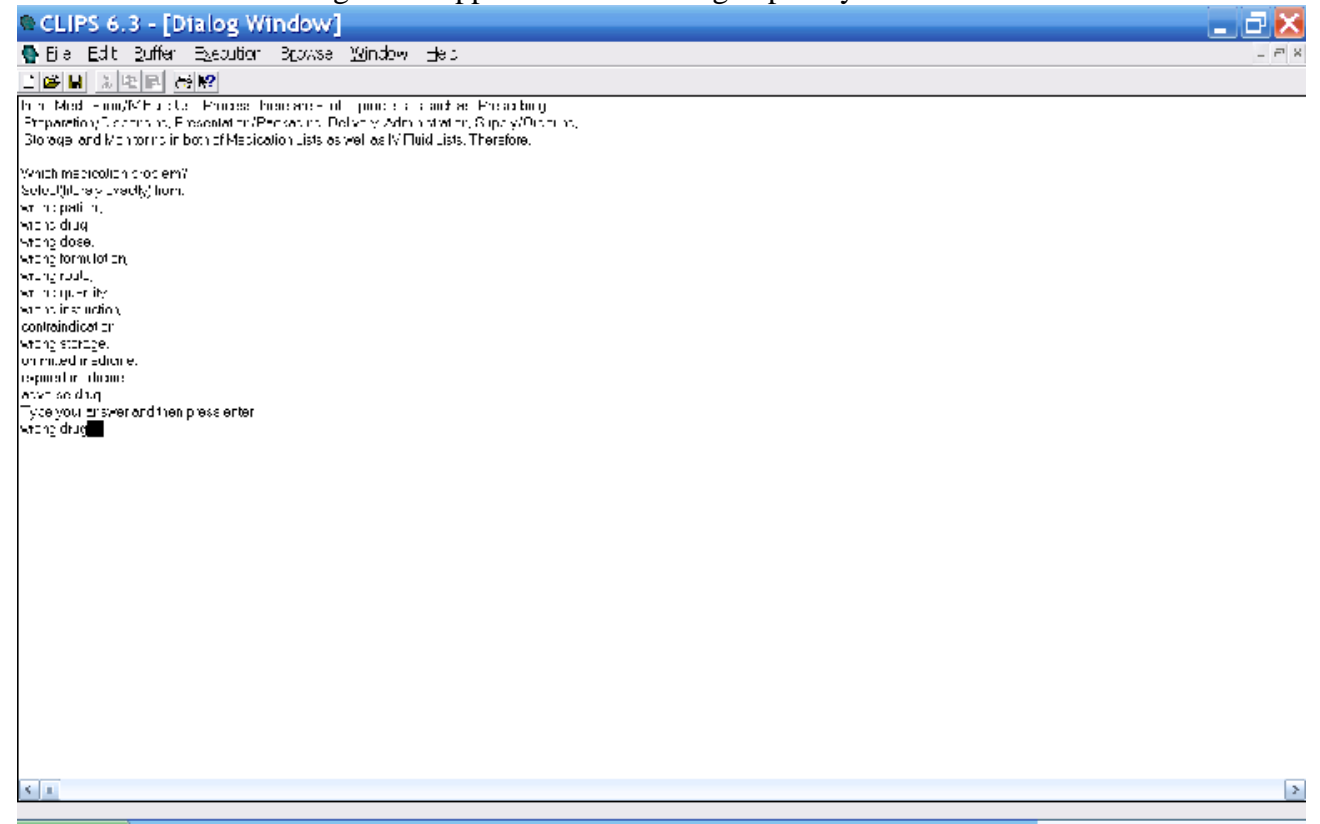

Figure 3. Input facts of a case study screen.

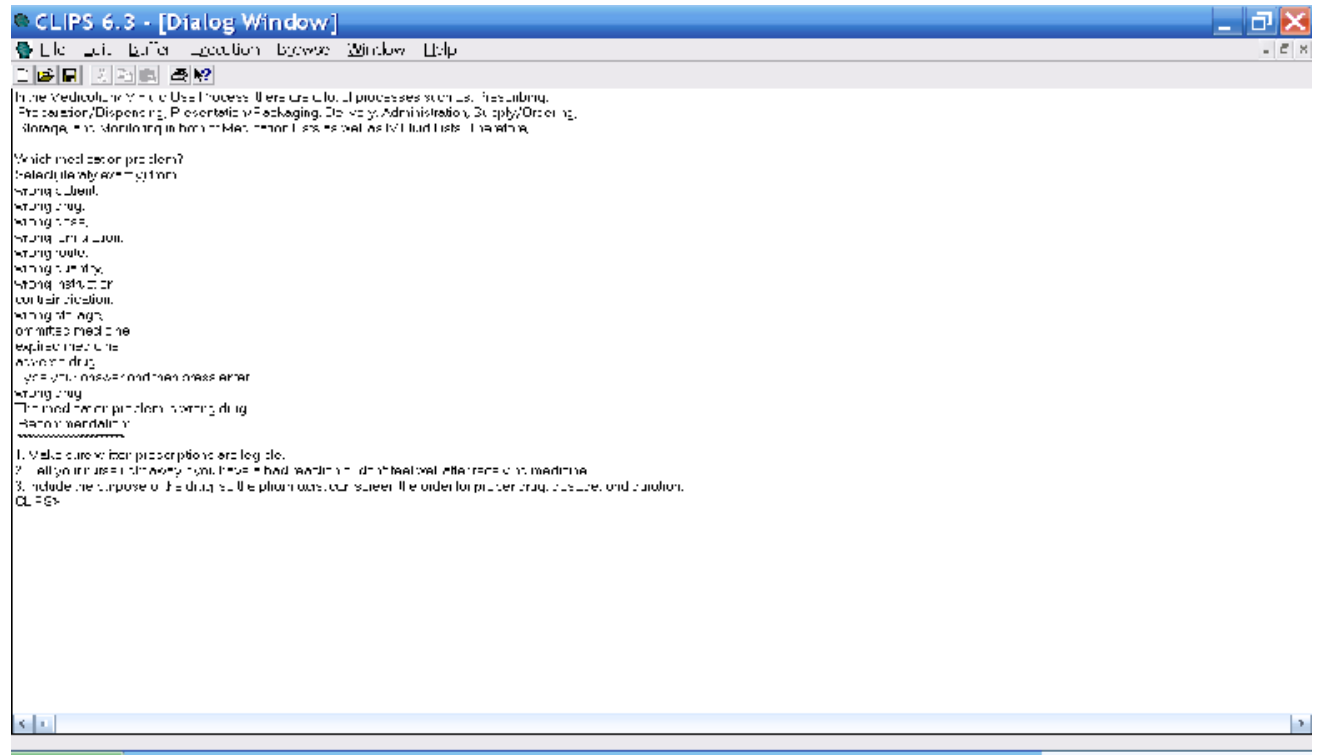

Figure 4. Output fact of a case study screen.

The following items are desiderata for responsible and appropriate medical errors classification expert systems: mechanisms of action; documentation of nomenclature; required training and skills for safe usage; mechanisms for obtaining help; intended uses and indications; contraindications and limitations to use; software version numbers and system function history; documentation of processes for building and maintenance of the system's knowledge base; 
expected duration of validity, existence of pre-programmed expirations, and suggested frequency of updates; record of previous evaluations; conformance to standards; and problem and error reporting.

The medical errors occurred by medical practitioners has received considerable attention in recent years. Yet there is a fundamental conflict amongst experts as to whether the number of deaths caused by these errors has been either under or over-estimated. Regardless, the number of deaths cause by such errors is frightening. Therefore, the nature of medical errors was analyzed, including errors in medications, treatment procedures, diagnosis, and clerical functions. This naturally led to our trial to extend the classification of medical errors. In addition we have discussed the possible past and future roles of computer software applications in reducing the rates of such errors. Developments and changes in software applications today offer unbelievable opportunities for "error-prevention and error-reduction." Hence we are optimistic that great progress can and will be made in this field.

\section{Acknowledgements}

This research was funded by King Abdel-Aziz for Science and Technology, Saudi Arabia, Grant AT\# 26-39.

\section{REFERENCES}

[1] Canadian Institute for Health Information. (2004) Report for Healthcare in Canada, Ottawa: Canada.

[2] Agency for Healthcare Research and Quality (AHRQ) (2008). The Fifth Annual Health Grades Patient Safety in American Hospitals Study. Obtained through the Internet: http://www.ahrq.gov/clinic/ptsafety/pdf/ptsafety.pdf, [accessed 7/10/2009].

[3] Baker, G., Norton, P., Flintoft, V., Blais, R., Brown, A. \& Cox, J. (2004) "The Canadian Adverse Events Study: the incidence of adverse events among hospitals in Canada" Canadian Medical Association Journal, Vol.170, pp.1678-1686.

[4] Goodman, F. (2004) "A fragmented patient safety concept: The structure and culture of safety management in health care" Nursing Economics, Vol. 22, No.1, pp.44-46.

[5] Fogarty, G. \& McKeon, C. (2006) "Patient safety during medication administration: The influence of organizational and individual variables on unsafe work practices and medication errors" Ergonomics, Vol. 49, No. (5-6), pp.444-456.

[6] World Health Organization, World Alliance for Patient Safety (2009). The International Classification for Patient Safety, Final Technical Report, Geneva: Switzerland.

[7] Meadows, M. (2003) "Strategies to reduce medical errors", FDA Consumer, Vol.37, No.3, pp. 20-27.

[8] Carayon, P., Gurses, A. \& Hundt, S. (2005) Performance obstacles and facilitators of healthcare providers, In: Korunka, C. and Hoffman, P. (ed.), Change and quality in human service work, Vol. 4. Munchen, Germany: Hampp Publishers.

[9] Rideout, K. (2006) Adverse medical event reduction technologies: identification of primary risk factors of adverse medical events using Artificial Neural Networks. Ph.D. thesis, Carleton University, Canada.

[10] Gavande, R. (1997). Medical Errors- How can they be reduced. Obtained through the Internet: http://www.engr.wisc.edu/wiscengr/Apr03/medical_errors.shtml, [accessed 9/6/2007].

[11] Morimoto T., Gandhi, T., Seger, A., Hsieh, T. \& Bates, D. (2004) 'Adverse drug events and medication errors: detection and classification methods' Quality \& Safety in Health Care, Vol. 13, pp. 306-314.

[12] Chang, A., Schyve, P., Croteau, R., O'Leary, D. \& Loeb, J. (2005) "The JCAHO patient safety event taxonomy: a standardized terminology and classification schema for near misses and adverse events" International Journal of Quality in Health Care, Vol. 17, pp.95-105. 
International Journal of Software Engineering \& Applications (IJSEA), Vol.4, No.6, November 2013

[13] Unruh, K. \& Pratt, W. (2006) 'Patients as actors: The patient's role in detecting, preventing, and recovering from medical errors' International Journal of Medical Informatics, Vol. 76, No. 1, pp.S236-S244.

[14] World Health Organization (2006). International Statistical Classification of Diseases and Related Health Problems. 10th Revision. Version for 2006 (ICD-10). Obtained through the Internet: http://www.who.int/classifications/icd/en/index.html, [accessed 10/6/2008].

[15] Espin, S. (2006) Error and reporting in surgery: exploring team and patient perceptions. Ph.D thesis, Department of Institute of Medical Science, University of Toronto, Canada.

[16] Institute for Safe Medication Practices Canada (2006). Canadian Medication Incident Reporting and Prevention System. Definitions of Terms. Obtained through the Internet:

[17] Crane, J. \& Crane, F. (2006), "Preventing Medication Errors in Hospitals through a Systems Approach and Technological Innovation: A Prescription for 2010" Hospital Topics, Vol. 84, No.4, pp.3-8.

[18] Elfering, A., Semmer, N. \& Grebner, S. (2006) "Work stress and patient safety: Observer-rated work stressors as predictors of characteristics of safety-related events reported by young nurses" Ergonomics, Vol. 49, No.(5-6), pp.457-469.

[19] Hogan, C. A. (2006) Pediatric Patient Safety: Factors pediatric nurses identify as contributing to medication administration errors. Ph.D. thesis, Nursing School, Loyola University, Chicago, Illinois, U.S.A.

[20] Hadi, W. (2013) "EMCAR: Expert Multi Class Based on Association Rule", International Journal of Modern Education and Computer Science, Vol.3, pp. 33-41.

[21] Kitporntheranunt, M., Wiriyasuttiwong, W., Development of a medical expert system for the diagnosis of ectopic pregnancy. Journal of the Medical Association of Thailand, 2010, 93 Suppl 2, pp. S43-49.

[22] Kumar, D.S., Sathyadevi, G., \& Sivanesh, S. (2011) "Decision Support System for Medical Diagnosis Using Data Mining”, International Journal of Computer Science Issues, Vol. 8, Issue 3, No. 1, pp. 147-153.

[23] Chang, R., (2011) "Interactive E-diagnosis: An Efficient Scheme for Medical Diagnosis Support System", Journal of Networks, Vol. 6, No. 5, 2011, pp. 713-720.

[24] Santosh, P. \& Patra, K. (2012) “Automatic Diagnosis of Diabetes by Expert System" International Journal of Computer Science Issues, Vol. 9, Issue 2, pp. 299-304.

[25] Gonz'alez, A., Ni no, J. \& Mayer, M.A. (2012) "Hernandez, G.A., Wilkinson, M.D., Analysis of a Multilevel Diagnosis Decision Support System and Its Implications: A Case Study”, Computational and Mathematical Methods in Medicine, Volume 2012, Article: ID 367345, pp. 1-9.

[26] Chi Fai David Lam, Kwong Sak Leung, Pheng Ann Heng, Chi Eung Danforn Lim \& Felix Wu Shun Wong, (2012) "Chinese Acupuncture Expert System (CAES)—A Useful Tool to Practice and Learn Medical Acupuncture” Journal of Medical Systems, Vol. 36, pp.1883-1890.

[27] Durkin, J. (1993) Expert Systems: Catalog of Applications, Akron: OH.

[28] Durinck, J., Coiera, E. \& Baud, R. (1994) "The role of knowledge based systems in clinical practice", In Barahona, P. and Christensen, J.(ed.), Knowledge and Decisions in Health Telematics - The Next Decade, (pp. 199-203) Amsterdam: IOS Press.

[29] Benner, P., Sheets, V., Uris, P., Malloch, K., Schwed \& Jamison, D. (2002) "Individual, practice, and system causes of errors in nursing” Journal of Nursing Administration, Vol. 32, No. 10, pp.509-523.[

[30] Santhiyakumari, N., Rajendran, P. \& Madheswaran, M. (2011) "Medical Decision-Making System of Ultrasound Carotid Artery Intima-Media Thickness Using Neural Networks" Journal of Digital Imaging, Vol.24, pp.1112-1125.

[31] Tomar, P.P., Singh, R. \& Saxena, P.K. (2012) “A Medical Multimedia based Clinical Decision Support System for Operational Chronic Lung Diseases Diagnosis and Training", International Journal of Computer Applications, Volume 49, No.8, pp. 1-12.

[32] Munirah, M., Rozlini, S.F., Chew, (2012) Design and Rules Development of Online Children Skin Diseases Diagnosis System, International Conference on Information and Knowledge Management (ICIKM 2012) IPCSIT vol.45, pp.272-276.

[33] Das, S., Chowdhury, S.R. \& Saha, H. (2012) “Accuracy Enhancement in a Fuzzy Expert Decision Making System Through Appropriate Determination of Membership Functions and Its Application in a Medical Diagnostic Decision Making System”, Journal of Medical Systems, Vol. 36, pp.1607-1620 
[34] CLIPS Software ver. 6.3. (2009). Obtained through the Internet: http://clipsrules.sourceforge.net/ \& http://sourceforge.net/projects/clipsrules/files/, [accessed 8/5/2009].

[35] Schmuller PC AI. (1991, 1992). "Expert System Shells at Work" series. Obtained through the Internet: http://www.pcai.com/web/ai_info/expert_systems.html, [accessed 1/12/2000].

[36] National Coordinating Council for Medication Error Reporting and Prevention. (1998) NCC MERP Taxonomy of Medication Errors, Rockville, MD: Office of the Secretariat, United States.

[37] Gawande A., Zinner, M., Studdert, D. \& Brennan, T. (2003) "Analysis of errors reported by surgeons at three teaching hospitals" Surgery, Vol.133, pp.614-21.

[38] Trucco, P. \& Cavallin, M. (2006) "A quantitative approach to clinical risk assessment: The CREA method" Safety Science, Vol. 44, pp. 491-513.

[39] National Health Service (2007). The National Reporting and Learning System National Patient Safety Agency. Obtained through the Internet: http://www.npsa.nhs.uk/nrls/reporting/, [accessed 10/6/2008].

[40] Runciman, W., Williamson, J., Deakin, A., Benveniste, K., Bannon, K. and Hibbert, P. (2006) 'An integrated framework for safety, quality and risk management: an information and incident management system based on a universal patient safety classification' Quality and Safety in Health Care, Vol.15, No.1, pp.i82-90.

[41] World Health Organization, Family of International Classifications Overview (2004a). Obtained through the Internet: http://www.who.int/classifications/en/, [accessed 9/6/2006].

[42] World Health Organization, Drug Dictionary (maintained by the Uppsala Monitoring Centre) (2004b). Obtained through the Internet: http://www.who.int/medicines/services/medicines_etools/en/, [accessed 9/6/2006].

[43] World Alliance for Patient Safety (2005). WHO Draft Guidelines for Adverse Event Reporting and Learning Systems. Geneva: World Health Organization (WHO/EIP/SPO/QPS/05.3).

[44] World Health Organization, Alliance for Patient Safety (2007a). Report on the Results of the WebBased Modified Delphi Survey of the International Classification for Patient Safety. Geneva: Switzerland.

[45] World Health Organization, Alliance for Patient Safety (2007b). Report of the WHO World Alliance for Patient Safety Meeting with Technical Experts from the South East Asian and Western Pacific Regions of the WHO, Tokyo: Japan.

[46] World Health Organization, Alliance for Patient Safety. (2008a). Report of the WHO World Alliance for Patient Safety Challenge Group Meeting - Validity Testing of the Conceptual Framework for the International Classification for Patient Safety, Geneva: Switzerland.

[47] World Health Organization, Alliance for Patient Safety (2008b). Report of the WHO World Alliance for Patient Safety Meeting with Francophone Technical Experts - Cultural and Linguistic Evaluation of the Conceptual Framework for the International Classification for Patient Safety, Paris: France.

[48] World Health Organization, Alliance for Patient Safety (2008c). Report of the WHO World Alliance for Patient Safety Meeting with Spanish and Latin American Technical Experts - Cultural and Linguistic Evaluation of the Conceptual Framework for the International Classification for Patient Safety, Madrid: Spain.

[49] Kopec, D., Kabir, H., Reinharth, D., Rothschild, O. \& Castiglione, J. (2003) 'Human Errors in Medical Practice: Systematic Classification and Reduction with Automated Information Systems' Journal of medical system, Vol. 27, No.4, pp.297-313.

[50] Miller, S. (2003) Medical Error Prevention. Health Studies Institute, Inc. Deerfield Beach: Florida, U.S.A.

[51] Halbach, J. \& Sullivan, L. (2003) A Curriculum Guide for Teaching Medical Students and Family Practice Residents, 3rd edition. Department of Family Medicine New York Medical College Valhalla: New York. 